\title{
CAPITALES DE LIDERAZGO EN LAS PROTESTAS TERRITORIALES: EL CASO DE DOS MOVIMIENTOS SOCIALES EN LA PATAGONIA CHILENA
}

IVÁN SANDOVAL ${ }^{\mathrm{a}}$, RODRIGO ASÚN ${ }^{\mathrm{a}}$, CLAUDIA ZÚÑIGA ${ }^{a} \&$ JAVIERA CORREA

\begin{abstract}
RESUMEN
La literatura reciente sobre movimientos sociales ha destacado el rol fundamental de los líderes o dirigentes en su emergencia y desarrollo y una de las más importantes discusiones al respecto se ha centrado en comprender la relación entre agencia y estructura en el contexto de las protestas colectivas. Utilizando herramientas conceptuales propuestas por Bourdieu (1986), se ha sugerido que el desempeño de los dirigentes de un movimiento social se puede entender a partir de su posición social en los distintos campos en que se mueven, determinada por el habitus y los tipos de capitales que poseen, y que para sortear obstáculos y reconocer y catalizar oportunidades políticas en el surgimiento y desarrollo de una movilización, se requiere la puesta en juego de tipos de capital distintos al económico. Desde una perspectiva cualitativa y analizando el discurso de los dirigentes de dos movimientos sociales territoriales ocurridos en el sur de Chile los años 2011 y 2012, buscamos comprender la importancia relativa de los distintos capitales de los dirigentes en el surgimiento, desarrollo y resultados de un movimiento social. Los movimientos territoriales son particularmente interesantes para este propósito, pues tradicionalmente se ha estudiado a dirigentes de movimientos de raíz funcional (i.e. estudiantes, obreros), étnica, de género y otros sociodemográficamente homogéneos, mientras que los movimientos territoriales tienden a convocar a personas de distintas edades, género, nivel socioeconómico, ocupaciones e ideologías, lo que complejiza la labor directiva y dificulta el logro de la confluencia de intereses, obligando a los dirigentes a desplegar y complementar sus redes, conocimientos, habilidades y recursos a fin de lograr el éxito del movimiento.
\end{abstract}

PALABRAS CLAVE: movimiento social, liderazgo, capital social, capital cultural, capital simbólico, acción colectiva.

\section{LEADERSHIP CAPITAL IN TERRITORIAL PROTESTS: THE CASE OF TWO SOCIAL MOVEMENTS IN THE CHILEAN PATAGONIA}

\section{ABSTRACT}

The recent literature on social movements has highlighted the fundamental role of leaders in their emergency and development. One of the most important discussions in this regard has focused

a Universidad de Chile, Avenida Ignacio Carrera Pinto 1045, Santiago, Nuñoa, Región Metropolitana, Chile.

\iesandoval@uc.cl; rasun@uchile.cl; cczuniga@u.uchile.cl; javiera.correa.u@gmail.com. 
on understanding the relationship between agency and structure in the context of collective protests. Using conceptual tools proposed by Bourdieu (1986), it has been suggested that the performance of the leaders of a social movement can be understood from their social position in the different fields in which they move, which in turn is determined by the habitus and the types of capital they own. In order to overcome obstacles and recognize and catalyze political opportunities in the emergence and development of a mobilization, the use of several types of capital other than economic is required. From a qualitative perspective and analyzing the discourse of the leaders of two territorial social movements that occurred in two regions of the south of Chile between 2011 and 2012, we seek to understand the relative importance of the different capitals of the leaders in the emergence, development and results of a social movement. Territorial movements are particularly interesting for this purpose, as it has traditionally been studied leaders based on functional (e.g. students, workers), ethnic, gender and other socio-demographically homogeneous movements, while territorial movements tend to summon people from different ages, gender, economic activities, ideologies and social levels, which complicates the work of leaders and makes it difficult to achieve the confluence of interests, forcing leaders to deploy and complement their networks, knowledge, skills and resources in order to achieve the success of the movement.

KEY WORDS: social movement, leadership, social capital, cultural capital, symbolic capital, collective action.

\section{INTRODUCCIÓN}

Si bien existe consenso respecto a la relevancia del rol que juegan los dirigentes en la emergencia y conducción de los movimientos sociales (Ganz, 2000; Morris \& Staggenborg, 2004; Nepstad \& Bob, 2006; Foldy et al. 2008; Robinson, 2009), no hay acuerdo respecto a su importancia relativa en relación con otros factores explicativos del éxito del movimiento. Algunas propuestas, como las teorías de movilización de recursos (McCarthy \& Wolfson, 1996; McCarthy \& Zald, 2001) y de proceso político (Smith \& Fetner, 2008; Robinson, 2009), otorgan mayor importancia a la estructura que a los dirigentes; otras, en cambio, destacan la capacidad de agencia y autonomía de los líderes o dirigentes, tanto en relación con sus capacidades estratégicas (Ganz, 2000), como en los procesos de construcción de sentido (Snow et al. 2014).

Una tercera vía para zanjar esta clásica discusión entre agencia y estructura consiste en emplear los conceptos de habitus y capital de Bourdieu para entender que ambas dimensiones de la vida social son planos de una misma realidad (Castón, 1996; Ávila, 2002; Gutiérrez, 2004; García, 2006; Rizo, 2006). Si el habitus se define como un "sistema de disposiciones duraderas y transferibles, estructurasestructuradas predispuestas a funcionar como estructuras estructurantes"
(Bourdieu, 1980, p. 86), se puede entender que los dirigentes han incorporado, producto de su historia y experiencias previas, disposiciones de larga data y difíciles de modificar, que condicionan al menos parcialmente su autonomía como agentes y que constituyen manifestaciones de la estructura social en su comportamiento. No obstante, la conducta cotidiana de los dirigentes también se ve influenciada por la particularidad de la contingencia, razón por la cual el habitus está en permanente reconstrucción, lo que implica que los dirigentes mantienen algunos niveles de autonomía frente a dicha estructura. En consecuencia, el concepto de habitus permite afirmar que las prácticas de los agentes son producto, tanto de estructuras sociales externas e incorporadas, como de ciertos niveles de autonomía.

Por otro lado, la posición social de los dirigentes depende en buena medida de la cantidad y tipos de capital que posean, y éstos a su vez adquieren mayor o menor relevancia en determinados campos de acción (Joignant, 2012; Husu, 2013). Entre los principales tipos de capital presentes en la teoría de Bourdieu se encuentran el cultural, el social y el simbólico (Nepstad \& Bob, 2006; Husu, 2013).

En los últimos años, se han realizado numerosas investigaciones utilizando este aparato teórico para el estudio del liderazgo en los movimientos sociales, enfatizando el concepto de 
habitus (Crossley, 2003; Haluza-Delay, 2008) y el de capital (Nepstad \& Bob, 2006; Bennister et al. 2014).

Crossley (2003), por ejemplo, considera que estudiar los movimientos sociales a partir de la teoría de las prácticas de Bourdieu permitiría resolver algunas de sus problemáticas clásicas. El concepto de habitus facilitaría comprender tanto las formas históricamente estables de participación de las personas en movimientos sociales, como la capacidad que tienen los dirigentes de modificar estas estructuras heredadas. Por su parte, HaluzaDelay (2008), a partir del estudio de movimientos medioambientales, plantea que la mutabilidad relativa de los habitus constituye una oportunidad para estos movimientos, ya que hace posible la transmisión de un habitus ecologista a personas no cercanas a esta ideología. Además, Husu (2013) utiliza los conceptos de campo, capital y habitus para estudiar distintos movimientos identitarios, planteando su relevancia para comprender la diversidad de espacios sociales que ocupan los participantes en esos movimientos y para postular que un requisito para su éxito es que sus dirigentes estén bien capacitados y cuenten con legitimidad frente a sus seguidores.

Ahora bien, los autores que se focalizan especialmente en el rol de los dirigentes de movimientos sociales tienden a utilizar el concepto de capital para explicar cómo estas personas logran hacer surgir, mantener activo y conseguir el éxito de un movimiento (Nepstad \& Bob, 2006; Bennister et al. 2014). En este marco, Nepstad y Bob (2006) postulan que los dirigentes o líderes poseen un capital de liderazgo, conformado por la suma de distintas formas clásicas de capital: cultural, social y simbólico. Los mismos autores plantean que si los líderes no poseen recursos económicos, pueden superar la falta de éstos mediante los capitales mencionados.

El capital cultural se refiere a los conocimientos y habilidades específicas que los líderes o dirigentes pueden desplegar en el marco de un conflicto o movimiento social (Nepstad \& Bob, 2006), y podemos distinguir tres tipos: localizado, universalista y transcultural. El capital localizado se refiere al conocimiento profundo de los imaginarios, valores, historia y formas de entender el mundo de las comunidades que buscan representar. El universalista hace referencia al conocimiento asociado a los valores, sentimientos y principios culturales a escala mayor, como la república o la nación, incluyendo a la población que se busca que apoye al movimiento y a los adversarios en el contexto nacional. El transcultural, en tanto, considera las habilidades para manejarse en distintos campos y lograr conectar con distintas audiencias (Nepstad \& Bob, 2006).

El capital social, por su parte, se entiende como las redes y relaciones de conocimiento, confianza y reciprocidad, tanto entre los propios líderes, como entre éstos y los participantes del movimiento. Es reconocido por diversos autores como un recurso fundamental para el éxito de un movimiento social, pues facilita la realización de acciones colectivas de diverso tipo (Ganz, 2010). Este capital se expresa de dos maneras: como lazos fuertes o como lazos débiles (Nepstad \& Bob, 2006; Ganz, 2010). Los primeros hacen referencia a vínculos de confianza, generalmente basados en relaciones cara a cara, mientras que los lazos débiles refieren a las vías de acceso de los dirigentes a la población o audiencias masivas (principalmente a través de los medios de comunicación), y a los vínculos formales o instrumentales con agentes en distintos campos. Para que un movimiento social tenga éxito, se plantea como necesario que los líderes o dirigentes hagan uso de ambos tipos de lazos (Ganz, 2010).

Finalmente, el capital simbólico refiere a los niveles de carisma, estatus y/o legitimidad social alcanzados por los líderes o dirigentes en un momento dado (Nepstad \& Bob, 2006).

Nepstad y Bob (2006) plantean que, haciendo uso de estos diferentes tipos de capital, los dirigentes de un movimiento social deben enfrentar tres desafíos cruciales: articular a distintas organizaciones sociales y sus militantes; obtener la adhesión de terceros (otras organizaciones y población externa a sus propias organizaciones); $y$, en muchos casos, manejar y sortear el ejercicio de la violencia por parte de agentes estatales y adversarios del movimiento.

En el presente estudio emplearemos este enfoque teórico para analizar dos campañas de protestas, evaluando el rol de los distintos capitales de los dirigentes en el afrontamiento de estas tareas, además de explorar su importancia en 
otras dos instancias cruciales para los movimientos sociales que no están en la conceptualización original de Nepstad y Bob (2006): la interacción con el adversario y el manejo de la conflictividad interna del movimiento. Por supuesto, el enfoque teórico que emplearemos no excluye otras posibles explicaciones del fenómeno, que podrían ser consideradas como complementarias o alternativas a la propuesta que planteamos.

En definitiva, en este trabajo se busca determinar hasta qué punto es posible interpretar el éxito o fracaso relativo de dos campañas de protestas a partir de la noción de capitales de liderazgo. En este marco, nuestro análisis se orienta por dos hipótesis complementarias. Por una parte, que en el abordaje de estas distintas tareas, en distintos momentos del movimiento algunos de los capitales tendrán un rol más relevante que otros. Por otra, que, en el contexto de movimientos sociales territoriales, conformados por coaliciones heterogéneas, es determinante la confluencia de capitales más que la posesión de manera individual por algún dirigente particular.

\section{Casos de Estudio}

Pese a ser la nación más larga del mundo, con 4.300 kilómetros de longitud, Chile es un país altamente centralizado, tanto con respecto a su concentración demográfica (más del $40 \%$ de la población vive en la región que aloja la capital nacional), como desde el punto de vista del poder político (Fløysand et al. 2010). Lo anterior quizá explica la poca frecuencia de desafíos regionalistas al poder central a lo largo de su historia (Salazar, 2005). Sin embargo, en el año 2010, por primera vez desde el fin de la dictadura de Augusto Pinochet (1973-1990), asumió un presidente de derecha democráticamente elegido (Sebastián Piñera), quien formuló una serie de promesas de campaña que no fueron cumplidas (Leupin, 2017), y cuya coalición política no tenía mayor raigambre en las organizaciones sociales nacionales o regionales. Lo anterior se constituyó en una oportunidad política para diversas organizaciones $y$ movimientos sociales del territorio, que en las décadas anteriores vieron muchas veces sofocados sus intentos de movilización por la presencia entre sus filas de muchos simpatizantes y adherentes a los partidos de la coalición política que gobernó Chile desde el fin de la dictadura militar. Es en este contexto donde se sitúan los movimientos regionalistas que estudiamos.

Existen numerosas aproximaciones para definir lo que sería un movimiento social, pero de acuerdo con Diani (1992), las distintas definiciones confluyen en tres aspectos fundamentales: existencia de redes de interacción informal; creencias compartidas y solidaridad entre los miembros de la comunidad movilizada; y la acción colectiva en torno a temas conflictivos. Los casos de estudio que hemos seleccionado para esta investigación cumplen con estos tres criterios.

Movimiento "No al alza del gas de Magallanes" (MM) del año 2011

La región de Magallanes es la más austral de Chile. Su nombre se debe a que la cruza el estrecho de Magallanes, paso marítimo de alta relevancia económica y turística debido a que comunica los océanos Pacífico y Atlántico. Se caracteriza por sus bajas temperaturas, fuertes vientos y torrentosas marejadas. Aunque ha transcurrido más de un siglo y medio desde la fundación de las primeras colonias exitosas en la zona, la región sigue siendo una zona de difícil acceso, pues numerosos glaciares impiden su comunicación por tierra con el resto del territorio chileno. Para llegar por tierra desde su capital regional (Punta Arenas) a la capital de la región con la que limita hacia el norte (Coyhaique) se debe viajar 1.200 kilómetros por Argentina, contexto en el cual las rutas marítimas y aéreas adquieren particular importancia.

La región cuenta con una amplia historia de luchas sociales. Ya a comienzos del siglo $\mathrm{XX}$ los colonos dedicados a la explotación forestal y ganadera entraron en conflicto con los grandes dueños de la tierra, que fueron apoyados por los funcionarios estatales. Este espíritu combativo se mantiene durante aquel siglo entre obreros y empresarios luego del descubrimiento de yacimientos de petróleo y gas, y tuvo como consecuencia el desarrollo de una región movilizada y activa, uno de cuyos hitos en la memoria colectiva reciente es haber sido escenario de una de las primeras manifestaciones masivas contra el dictador Augusto Pinochet (el 
"Puntarenazo"), con motivo de su visita a la región en 1984 (Cavallo et al. 2008).

Según nuestra revisión sistemática de prensa, el movimiento "No al alza del gas de Magallanes" tiene su inicio en diciembre de 2010, cuando el gobierno aprueba un proyecto de ley que permite un alza del precio del gas en la región de casi un $17 \%$. Con una temperatura media en invierno de $2,4{ }^{\circ} \mathrm{C}$ (Butorovic, 2015) y con el gas como principal medio de calefacción residencial, esta alza afectaba de modo importante a toda la población. En respuesta, muchas personas salieron a la calle a manifestar su descontento y se formó una coalición de organizaciones sociales de la región, cuya vocería y coordinación fue asumida por una "Asamblea Ciudadana", integrada por dirigentes territoriales, sindicales, de funcionarios públicos, de pequeños empresarios y de organizaciones de derechos humanos, entre otros. Se paralizaron todas las actividades económicas y se bloquearon las vías de acceso a la región por medio del control de caminos, puertos y aeropuertos. Muy rápidamente, la Asamblea recibió el apoyo de la totalidad de los actores políticos de la región, incluyendo aquellos pertenecientes a los partidos que integraban el gobierno nacional.

Si bien la duración del movimiento fue breve (13 días), se llevaron a cabo 37 eventos de protesta, mayoritariamente pacíficos, disruptivos y masivos, como la realización de marchas, concentraciones, levantamiento de barricadas y bloqueo de caminos. No hubo intervención de fuerzas policiales y el gobierno optó por el diálogo, cuyo producto fue un acuerdo que estableció un alza del gas de solo el 3\%, acompañado por un importante número de subsidios a las familias más pobres de la región. Esta solución fue considerada un éxito por la mayoría de los dirigentes, aunque dejó disconforme a algunos que esperaban una anulación total del alza de precio y a aquellos que consideraban que se había desperdiciado la oportunidad de plantear otras demandas regionales históricas de diversa indole (vivienda, salud y trabajo principalmente).

Movimiento "Aysén, tu problema es mi problema” (MA) del año 2012

La región de Aysén se ubica al norte de Magallanes y aunque está conectada por vía terrestre al resto del país, su gran tamaño y accidentada geografía (plena de ríos, montañas, islas, glaciares y bosques) la aíslan externamente y dificultan sus comunicaciones internas.

A diferencia de la región de Magallanes, la de Aysén tuvo una colonización más bien tardía (años 70 y 80 del siglo XX), producto de la decisión gubernamental de poblar la región con colonos a cambio de la cesión de terrenos, utilizados principalmente para la explotación de ganado ovino (Arenas, 2009). La dispersión entre los diversos asentamientos poblacionales incluso hoy genera problemas en aspectos como la salud o la educación, lo que tiene como consecuencia tasas de alfabetismo y escolaridad inferiores a la media nacional (CASEN, 2013).

Se trata de una región sin mucha historia de movilizaciones, a excepción de aquellas desarrolladas por un fuerte gremio de pescadores artesanales, quienes tienen un importante rol en la identidad y economía regional (casi el 20\% del PIB local; Consejo Nacional de la Cultura y las Artes, 2015). El origen de las movilizaciones de 2012 estuvo en la confluencia de un importante número de demandas regionales y el "efecto demostración" de otras movilizaciones que ocurrieron en Chile en esos años (Radovic, 2013). Entre ellas, el movimiento de Magallanes de 2011, movilizaciones nacionales contra el proyecto hidroeléctrico HidroAysén ("Patagonia Sin Represas") y el movimiento estudiantil chileno del mismo año, junto a protestas locales contra la ley de pesca que sería aprobada el año 2012.

Esas manifestaciones crearon el terreno para que algunos dirigentes sociales comenzaran a coordinarse más eficazmente y adquirieran visibilidad. El movimiento incluyó 99 eventos de protesta en un período de 56 días (según nuestra revisión sistemática de la prensa), paralizando a la región desde febrero a marzo de 2012 y recibiendo una violenta respuesta de parte del Estado. A diferencia del MM, este movimiento planteó al gobierno un extenso petitorio que pasó de 11 demandas iniciales a un listado de 71 demandas heterogéneas. Después de más de un mes y medio de movilizaciones y fuerte represión policial, un grupo de dirigentes viajó a la capital y pactóó el fin de aquéllas obteniendo concesiones que fueron consideradas insuficientes por la mayoría de los dirigentes. 
En definitiva, se analizaron dos campañas de protestas que, a pesar de tener una base de participantes y reivindicaciones marcadamente regionalistas, presentan procesos de emergencia y desarrollo disímiles. La evaluación de sus resultados, además, es materia de discrepancia tanto para quienes participaron como para la población en general. Mientras la movilización de Magallanes fue considerada exitosa por la mayor parte de sus participantes, la ocurrida en Aysén generó un escenario de frustración generalizada entre los dirigentes. En este marco, nuestro análisis apuntó a dilucidar el rol y la importancia relativa de los distintos tipos de capital puestos a disposición por cada conjunto de dirigentes en los distintos momentos y tareas de la movilización.

\section{MÉTODO}

\section{Enfoque}

Se optó por un enfoque cualitativo, con el objetivo de conocer en mayor profundidad, y desde la perspectiva de los propios actores, el rol que jugaron los dirigentes en los distintos procesos del movimiento. A partir del análisis de sus reflexiones sobre las dinámicas intra e inter organizacionales, las estrategias que desplegaron para hacer frente a los desafíos del trabajo dirigencial y los aprendizajes que extraen a partir de lo anterior, fue posible identificar la movilización y puesta en común de distintos tipos de capital.

\section{Técnicas de producción de información}

Se empleó principalmente entrevistas semiestructuradas, con el fin de levantar los distintos discursos de los dirigentes de cada movimiento, y abordar aspectos que pudieran surgir durante las entrevistas y no se hubieran tenido en consideración a priori. Por ello, la pauta sirvió para guiar la conversación, pero se adaptó a cada entrevistado. Las entrevistas se llevaron a cabo durante el año 2014.

\section{Participantes}

Se utilizó un muestreo teórico intencionado, cuyo objetivo fue obtener una muestra lo más exhaustiva posible del total de organizaciones y dirigentes de cada movimiento, para así poder acceder a las distintas experiencias, posiciones y trayectorias directivas que convergieron en ellos.

El principal criterio de inclusión fue que los entrevistados hubieran tenido un rol activo en la conducción de su respectivo movimiento, contando con la representación legítima de los militantes de su organización. Además, se buscó heterogeneidad en el género, la edad, el tipo de organización, la orientación política y la participación previa en otros movimientos sociales de los potenciales participantes. En ambos movimientos, los dirigentes demostraron tener una buena disposición a participar en la investigación, por lo que nadie rechazó ser entrevistado.

Se entrevistó a ocho dirigentes del MM y once del MA. Pese a nuestros esfuerzos por obtener un grupo heterogéneo, debido al tipo de liderazgo de ambos movimientos, los entrevistados en su mayoría fueron hombres (14 de 18), quienes en gran parte tenían experiencia de participación en las movilizaciones previamente ocurridas en sus respectivas regiones. Debido a que los participantes pertenecen a comunidades pequeñas, en la presentación de resultados no se informará ningún dato sociodemográfico ni de la organización que representan, para proteger la confiabilidad.

\section{Técnica de análisis}

Se utilizó el análisis de contenido categorial pues éste apunta a conocer y describir los contenidos latentes de las comunicaciones de los sujetos. Para ello se categorizaron los discursos de los participantes en función de los constructos descritos en el marco teórico, lo que nos permitió conocer los distintos tipos de capital que debieron emplear para desarrollar su cometido como dirigentes de un movimiento social.

\section{RESULTADOS}

Para la organización de los resultados, éstos se agruparán en función de cuatro grandes metas o desafíos que deben enfrentar los dirigentes de un movimiento social: lograr que surja un movimiento, lograr la adhesión de terceros, interactuar con el adversario y manejar la conflictividad interna. 
Para ejemplificar estos resultados se presentan citas textuales de los entrevistados, identificando el movimiento al que pertenecen y el número de entrevista.

\section{Emergencia del movimiento}

Nepstad y Bob (2006) proponen que la articulación exitosa en un movimiento social de organizaciones preexistentes depende de que sus líderes posean capital cultural localizado, capital social fuerte y débil, y capital simbólico. Ello coincide parcialmente con nuestros resultados, pues éstos dejan en evidencia el importante rol de los capitales social fuerte y cultural localizado, en desmedro del capital social débil y el simbólico. Esta diferencia podría deberse a dos factores. Por una parte, a que en los movimientos territoriales la población que se busca movilizar es primeramente aquella que reside en el territorio en cuestión, más que la del ámbito nacional. En segundo lugar, a que la legitimidad de los dirigentes en buena medida deriva de su calidad de representantes del amplio espectro de organizaciones sociales de la región. En este sentido, es un elemento que se asume como evidente, no es cuestionado, $y$, por ende, no necesita ser puesto en juego por las dirigencias para validar la emergencia de los movimientos.

El discurso de los entrevistados muestra la relevancia que tuvo en ambos movimientos la existencia de procesos previos de articulación entre algunas de las organizaciones que formarían el núcleo central, lo que se puede entender como la conformación de capital social fuerte. En el caso del MM, se menciona la aparición del movimiento "Magallanes se levanta" el año 2009, en el cual se articularon varias organizaciones sociales (juntas de vecinos y uniones comunales, organizaciones sindicales, de jubilados $y$ deportivas) para plantear demandas regionales. El mismo año, dirigentes de asociaciones gremiales vinculadas a la pequeña, mediana y gran empresa regional habían conformado el "Comité de Defensa de Magallanes", que adquirió experiencia en movilizaciones contra el alza del precio del gas. En el caso del MA, las organizaciones de pescadores artesanales habían apoyado previamente a organizaciones sociales coordinadas contra la instalación de un gran proyecto hidroeléctrico en la región (movimiento "Patagonia Sin Represas") en el año 2011. Además, también el año 2011 las secciones regionales de las dos organizaciones sindicales más importantes del país (Central Unitaria de Trabajadores -CUT- y Agrupación Nacional de Empleados Fiscales -ANEF-), habían actuado juntas por demandas laborales y se habían reunido con organizaciones vecinales, estudiantiles y ambientalistas enarbolando demandas regionales. Todo esto permitió la construcción de redes que fueron activadas en el momento en que se presentaron oportunidades políticas.

Magallanes. E.5. "Las organizaciones sociales ya, con mucha antelación, habiamos estado haciendo una suerte de reuniones para ir conformando un movimiento que se preocupe, justamente, de las demandas sociales de nuestra región".

Aysén. E.3. "Al principio fueron unas reuniones de grupos que teníamos algunas cosas en común, grupos bien activos que teníamos experiencia en calle, y en el fondo habíamos hecho algunas acciones de calle, otros habían hecho algunas acciones de fuerza, en el caso de los pescadores, pero nos conocíamos. Por lo menos yo conocía a varios del sector de los pescadores artesanales, la ANEF y la CUT con la cual también habíamos compartido calle, y Patagonia Sin Represas. Esos fueron como los tres grupos que decidieron conversar".

También se puede entender la articulación y legitimación previa como fuente de capital cultural localizado, en tanto generó conocimiento de las distintas organizaciones que se encontraban activas en el territorio y de sus atributos. Entre ellos, quiénes las lideraban, cuáles eran sus tendencias políticas, cuáles eran sus posturas frente a diversas problemáticas, sus formas de trabajo y su experiencia y competencias. Esto último fue particularmente relevante para la identificación de "organizaciones clave" para el éxito del movimiento. En el caso del MM, los dirigentes se concentraron en obtener el apoyo de los gremios del transporte, como elementos centrales para conseguir la paralización de la región, toda vez que la importación de bienes es central para su normal funcionamiento. En el caso del MA, los dirigentes coincidieron en el reconocimiento de dos organizaciones clave: los gremios de pescadores 
artesanales y la organización ambientalista "Patagonia Sin Represas". Los primeros poseían experiencia de movilizaciones y enfrentamiento con la policía, mientras que la segunda contaba con conocimiento académico, experiencia en el campo judicial y una red de contactos en toda la región (a diferencia de los pescadores, por estar radicados en zonas costeras).

Magallanes. E.2. "Estábamos trabajando para hacer un buen paro, que podía significar 24 horas de cortes de carreteras y de obstrucción del sistema, pero no sé si íbamos a aguantar 2 días (...). Se contactaron camioneros, se contactaron distintos sectores, decidimos cortes de carretera en algunos puntos estratégicos".

Magallanes. E.1. "Hubo un momento, durante el paro del gas, cuando se reafirma la participación de los camioneros, que eran los que aseguraban la paralización total del transporte en la ciudad y en la región".

Aysén. E.3. "Yo conocía que los viejos son fuertes, que son de tiro largo, como se dice acá, $y$ que pueden llevar una movilización fuerte $y$ mucho tiempo. Y el que estuvieran los otros grupos [organizaciones sociales] dispuestos a conversar, estaba como el escenario y el ambiente político como para que se pudiera dar eso".

Aysén. E.11. "Entre la gente que estaba participando en eso había gente que sabía de haber participado en batallas campales con carabineros, había quienes sabían hacer y difundir la noticia, y había quienes podian encantar a la gente, y era como buscar las herramientas y los mecanismos para lograr levantar un movimiento social".

El capital cultural localizado también permitió a los dirigentes de ambos movimientos determinar la forma de trabajo más efectiva para legitimarse ante el conjunto de las organizaciones sociales y la ciudadanía. La pluralidad política y social de los dirigentes, junto con la deslegitimación de la política tradicional, acentuaron la necesidad de presentarse como representantes ciudadanos y voceros de organizaciones sociales, y dejar de lado cualquier militancia partidista. En el caso del MM, ello implicó formar una estructura asamblearia horizontal y no tener representantes con mayores niveles de poder resolutivo. En el caso del MA, la horizontalidad se dio en la articulación de demandas, pero no en las jerarquías representativas, pues surgió un líder carismático que terminó por concentrar el poder resolutivo y de negociación.

El capital cultural localizado de los dirigentes también se expresó en lo que refiere al conocimiento de los problemas y luchas históricas de la región, y de las opiniones y sentimientos de la ciudadanía respecto a esos problemas. Sobre todo, estaban conscientes del amplio y transversal rechazo ciudadano al "centralismo" del Estado. Este fenómeno es identificado, por una parte, como el abandono de la región en lo referente al desarrollo de inversiones y políticas públicas de salud, trabajo, educación y conectividad, lo cual es especialmente sentido en regiones donde el aislamiento encarece productos esenciales para la vida cotidiana. Por otra parte, el centralismo es asociado a la exclusión de sus habitantes de las instancias de toma de decisiones sobre la explotación y uso de sus recursos locales. Por tanto, los dirigentes podían prever una adhesión numerosa de la ciudadanía a los movimientos, y encontraron un referente discursivo aglutinante al cual recurrir.

Magallanes. E.1. "Hay una suerte, también, como de orgullo regionalista frente a un Estado central que ha sido mezquino, egoísta con la región, que ha mirado en menos a la región. Pero esto no lo dejamos en la queja, no es un llanto ni es un quejoneo de que a nosotros no nos dan lo que nosotros merecemos, sino que hay un sentido de lucha, de enfrentar al centralismo con movilización social".

Aysén. E.5. "Ahora sí hay un elemento aglutinante que es el hecho de que hay mucha percepción en la región de que al aisenino siempre se lo han cagado de afuera, que, porque somos pocos, porque vivimos lejos, por millones de motivos, el centralismo ha tomado decisiones y ha perjudicado Aysén".

Finalmente, en cuanto al capital cultural universalista, la mayoría de los dirigentes conocía la eficacia de las estrategias de manejo de conflictos y movimientos sociales utilizadas por la coalición política de centro izquierda que gobernaba Chile anteriormente ("Concertación de Partidos por la Democracia"), consistentes en utilizar a sus bases militantes insertas en organizaciones sociales para evitar las protestas o terminarlas rápidamente 
aceptando concesiones menores. Por ello, la existencia de un gobierno de derecha sin presencia en las organizaciones sociales y sin experiencia en el manejo de los conflictos sociales fue correcta y explícitamente identificada como una importante oportunidad política.

Magallanes. E.2. "La Concertación por 20 años sofocó todos los movimientos habidos y por haber, y a nosotros la encachada nos duraba 30 minutos, porque te daban el $50 \%$ de la solución y eso ya era algo".

Aysén. E.7. "Además que los políticos en la Concertación son habilosos. (...) Es $m$ esos no son muy políticos, es mcha de una librero la comisi gador determinado.ser 20 dacionalás, yo les digo, el movimiento de Aysén se hizo porque estaba la derecha [en el gobierno], porque si hubiera estado la Concertación, el movimiento no logra llegar a ser movimiento, porque lo habrian infiltrado".

\section{Adhesión de terceros}

Se ha reconocido que la obtención de apoyo de personas y organizaciones externas al movimiento puede incrementar sus probabilidades de éxito al facilitarle el acceso a recursos (dinero, conocimientos, redes o legitimidad) y aumentar la presión sobre sus adversarios (Nepstad \& Bob, 2006). En el caso de los movimientos sociales territoriales son dos los principales actores externos a seducir: las organizaciones sociales que no se adhirieron inicialmente al movimiento y la ciudadanía que no es parte de dichas organizaciones, tanto aquella que habita en la región como la del resto del país.

Tal como proponen Nepstad y Bob (2006), nuestros resultados indican que la tríada de capital cultural localizado, social y simbólico, es efectivamente utilizada por los dirigentes de ambos movimientos.

En términos de capital cultural localizado, fue clave en el diagnóstico de la apertura de una oportunidad política a partir de la conjunción de factores coyunturales e históricos. En ambos casos, el rechazo transversal al centralismo se leyó como un factor movilizador y aglutinante, y fue además estimulado por acciones de las autoridades. El incumplimiento de promesas de campaña y algunas declaraciones despectivas de las autoridades hacia la población provocaron un repudio generalizado transversalmente.

Magallanes. E.1. "Lo que va a acentuar lo que podríamos denominar la indignación de la ciudadanía, es la reacción del Gobierno. La torpeza de la intendenta, que dice "yo no creo que sea posible evitar el [alza del] precio del gas, porque si no el gas se nos va a terminar'. La torpeza de la vocera de gobierno, Ena von Baer, que justifica el alza del gas y que hace una alusión muy poco feliz cuando habla de que 'ahora a los magallánicos se les va a terminar la fiesta'. La torpeza del Ministro Raineri, de Energía, que justifica también el alza del gas".

Aysén. E.9. "Parece súper irreal lo que te cuento. Funcionarios públicos, obreros, pescadores, estudiantes, dueñas de casa, todos, todos dándole, algunos en un puesto, otros en otro, pero todos dándole. Gente de derecha, mucha gente de derecha, te diré. Entonces, claro, debe ser súper difícil imaginarlo, el cómo se llegó a eso. Pero hay razones objetivas también. El 2011 se cerró con muchas respuestas negativas para un montón de gremios. Hay que recordar que Piñera se instala en el poder inflando una expectativa del porte de un rascacielos. Entonces, a muchas organizaciones se les prometió, se les aseguró que se le iban a mejorar sus condiciones, (...) y a la hora de ir a cobrar el compromiso, el 2011 no pasó nada".

En Aysén, la heterogeneidad de las demandas regionales hizo que los dirigentes tuvieran que extremar sus habilidades retóricas, no solo para motivar la participación, sino también para instalar en el discurso público las distintas problemáticas que el movimiento buscaba solucionar, y convencer a las personas de que éstas les afectaban de manera transversal en sus vidas cotidianas. Fue a partir de este esfuerzo que crearon la consigna "tu problema es mi problema".

Este tipo de capital cultural también fue importante para ayudar a los dirigentes a decidir a qué medios de comunicación acudir para difundir su mensaje, dadas sus líneas editoriales y popularidad entre la población local. En complemento, la existencia de vínculos con medios de comunicación locales -especialmente la radio-, es decir, capital social débil, permitió 
sortear con éxito el bloqueo de los medios nacionales, informando y convocando a distintos eventos y protestas, incluso en los sectores más apartados.

En cuanto a la adhesión de organizaciones externas, en ambos movimientos parece haber sido determinante el capital social fuerte de los dirigentes en forma de redes de trabajo y colaboraciones previas con otras organizaciones locales, toda vez que se emplearon aquellas redes para convocar y movilizar a otras organizaciones con las que cada uno tenía contacto.

Con relación al capital simbólico, los dirigentes contaban con cierto reconocimiento entre la ciudadanía y eran percibidos por ésta como militantes políticamente heterogéneos, comprometidos con sus representados y la región, y no con partidos políticos nacionales, lo que les daba mayor legitimidad. En Magallanes, los dirigentes limitaron la participación en la Asamblea de personas reconocidas como militantes de partidos políticos, lo cual se puede entender también como una aplicación de capital cultural localizado en la toma de decisiones. En Aysén, a lo anterior se sumó el reconocimiento público de algunos dirigentes por su participación en protestas previas y su presencia en medios de comunicación locales, lo que fue particularmente fuerte en el caso de un dirigente de los pescadores que se posicionó como líder carismático.

Aysén. E.2. "La gente le pasaba las guaguas pa' que las abrace, así como un semidiós, un héroe, así como que le pasaban las guaguas. Y, sí po, había un gran poder de convencimiento".

\section{Interacción con el adversario}

En los movimientos sociales territoriales es particularmente importante analizar la interacción con los adversarios, pues en este proceso la labor de los dirigentes es clave. Deben leer el ambiente político, evaluar las fortalezas y debilidades propias y del adversario, escoger la mejor vía de interacción con éste y definir los mecanismos de presión, especificando la cantidad de esfuerzo que se destinará a cada uno y quiénes se harán cargo de ellos. Dada la heterogeneidad de los dirigentes $y$ organizaciones que componen los movimientos territoriales, distintos tipos de capital pueden ser puestos en juego dependiendo del modo en que se lleve a cabo la interacción con el adversario: negociación, resistencia, oposición violenta, entre otras.

En ambos movimientos parece haber sido relevante la experiencia de participación de los dirigentes en movilizaciones anteriores. Esa fuente de capital cultural localizado y universalista les permitió conocer los atributos de las diversas organizaciones y ciudadanía local, así como el modo de acción de los representantes del gobierno.

En el MM, a partir de aquel conocimiento se optó por enfocar la interacción con el adversario en dos frentes. En primer lugar, se participó en una mesa de negociación con ministros del gobierno, a la cual se decidió que asistiera la gran mayoría de los dirigentes de la Asamblea Ciudadana de Magallanes, y no delegar la función de representación a un grupo más reducido. Al mismo tiempo, se apoyó la realización de protestas callejeras de carácter pacífico, muchas de las cuales eran promovidas autónomamente por la ciudadanía. De esta manera, mantuvieron la presión sobre los adversarios, sin desgastar mayormente a los dirigentes.

El MA, por su parte, también tuvo dos frentes de interacción con sus adversarios. Por un lado, una mesa de negociación con los ministros y enviados de gobierno, que a diferencia del $\mathrm{MM}$, estuvo a cargo de un conjunto reducido de dirigentes que actuaron como voceros. Por otro, protestas callejeras de carácter más violento, por lo cual el movimiento enfrentó la represión de fuerzas policiales enviadas por el gobierno.

En la conducción de la negociación la posesión de capital cultural universalista, en tanto conocimiento de los atributos de los representantes del gobierno (estilos de negociación, fortalezas y debilidades), parece haber sido muy importante para ambos movimientos. En Magallanes, ello permitió a los dirigentes pronosticar también un estilo de negociación poco flexible $y$ autoritario por parte de los representantes del gobierno y explotar aquellas debilidades. Varios entrevistados señalaron que esperaban que la falta de experiencia política de los ministros, que en su mayoría provenía del mundo empresarial, se tradujera en un estilo de negociación torpe, pues estaban acostumbrados a operar de manera más bien autoritaria. 
Magallanes. E.8. "Piñera cuando colocó sus ministros y todo, colocó mucha gente supuestamente de excelencia, pero resulta que ellos en el tema social, como grandes empresarios, porque son patrones de fundo, no les gusta negociar. Entonces, por eso fue que esto fue tan caótico acá".

Magallanes. E.2. "(...) cuando le poníamos al frente a dos dirigentes de la pesca, el ministro se quería morir. Y los dirigentes de la pesca lo único que sabían es que el gas no podía subir acá, punto, nada más. Y él se desgastaba 1 hora, 2 horas en tratar de explicar. Nosotros mirando por la ventana".

Además, gracias al capital social fuerte y débil de los dirigentes, en ambos movimientos se pudo acceder a conocimientos técnicos y asesorías. En Magallanes, fue clave la ayuda de sindicatos de la Empresa Nacional del Petróleo y de empresarios regionales, para rebatir argumentos respecto del agotamiento del gas en la región y demostrar los privilegios que poseía una empresa privada apoyada por el gobierno. En Aysén, en tanto, se sumó el apoyo y asesoría de dirigentes nacionales de las organizaciones de trabajadores cuyas dirigencias regionales participaban activamente en la movilización.

Ahora bien, el conocimiento del adversario, sumado al conocimiento de las características del movimiento y la ciudadanía, es decir, capital cultural localizado, fue particularmente clave en la definición de las formas de presionar a las autoridades. Los dirigentes del MM coincidieron en la importancia de mantener la paralización total de la región y sostener la cohesión interna. Para lograr lo primero, sabían que era vital el corte de rutas en puntos específicos, bloqueando el tránsito de bienes y personas hacia y desde la capital regional, Punta Arenas. Además, tenían claro que la escasez general que provocaría la paralización les daba poco tiempo para lograr sus objetivos, por lo que decidieron enfocarse exclusivamente en la demanda de congelar el precio del gas, y no promover otras peticiones sectoriales, que no generaban una adhesión general de la ciudadanía.

Magallanes. E.2. "Teníamos esa lectura nosotros, había que aprovechar de poner otros temas, pero también corríamos el riesgo de que se enredara el asunto. Entonces, nos fuimos por el lado de la energía y dijimos 'mira, aquí más allá de que no queremos que haya alza del gas, además queremos que ENAP se transforme en la gran empresa de energía, acá hay la posibilidad de energías alternativas, de explorar eso', pero, en definitiva, los tiempos no daban para más que el debate diario, cotidiano, del guarismo del gas".

En Aysén, en cambio, se tenía certeza de que la presión al adversario dependía de la presencia en las protestas callejeras, tales como barricadas y cortes de ruta, que seguramente encontrarian una respuesta violenta por parte de las fuerzas policiales. Por ello, se entregó la conducción de aquella tarea al gremio de pescadores artesanales, que era la organización con más experiencia y habilidades tácticas en esta materia. También fue crucial el conocimiento de que la ciudadanía regional podría soportar un período prolongado de escasez generada por la paralización, pues la resistencia a las privaciones es parte de la identidad regional. No obstante, incluso teniendo esto en cuenta, las negociaciones se prolongaron varias semanas más de lo esperado, por lo que algunos dirigentes terminaron cediendo en varios puntos a favor del gobierno.

Aysén. E.4. "El tema de no tener qué comer, porque no había más comida, hay gente que lo vive todos los meses. Lo que pasa es que ahora le faltó a alguien que tiene para comer y no le llegaron sus camarones, y no tenía cigarros, y no podía comer lo que comía todos los días, a ese le dolió harto, pero la gente de las poblaciones esta apretura la vive todos los meses y durante años".

De tal manera, mientras en Magallanes los dirigentes en su gran mayoría se manifestaron satisfechos con lo obtenido, aun cuando algunos lo consideraban insuficiente, en Aysén la evaluación general fue de insatisfacción. En esta situación puede haber incidido, entre otras cosas, la ausencia o falta de movilización de dos tipos de capital entre los dirigentes del MA. En primer lugar, es posible que la falta de experiencia de los dirigentes en el manejo de demandas disímiles, lo cual se puede entender como capital transcultural en el ámbito del activismo (Van Dyke \& Dixon, 2013), les impidió distinguir entre aspiraciones prioritarias y secundarias, conspirando contra la unidad del movimiento. La ausencia de una demanda central 
exigió a los dirigentes del MA la difícil capacidad de integrar las peticiones de los diversos sectores de la ciudadanía sin aumentar el pliego de demandas. $\mathrm{Al}$ comienzo se logró a través de un proceso de deliberación, pero luego se abandonó, por lo que finalmente el petitorio presentado a las autoridades terminó conformado por 71 puntos bastante heterogéneos, pocos de ellos compartidos por todos los dirigentes.

En segundo lugar, la falta de capital social fuerte entre los dirigentes, en términos de confianza entre pares, les dificultó mantener una estructura y controlar los personalismos. Con el avance de las negociaciones, la representación y poder resolutivo del movimiento se concentró en un dirigente carismático, quien terminó viajando a Santiago, la capital nacional y sede del gobierno, para negociar el fin de las movilizaciones. Esta situación quebró las relaciones al interior del movimiento y precipitó su término. Ello queda de manifiesto en las palabras de algunos dirigentes al referirse a quienes finalmente negociaron con el gobierno el término de las movilizaciones.

Aysén. E.7. "Nosotros sabiamos que estos compadres, ya por experiencias de otros movimientos, ya nosotros sabíamos que estos compadres eran traidores, que éstos se vendian, negociaban, y nosotros no negociábamos, éramos como los duros de la lucha".

Aysén. E. 5. "La primera vez que cambió una letra de la propuesta, fue un vendido. $\mathrm{O}$ sea, para todos, para algunos pescadores, 'no, se vendió, nos cagó', porque tenían experiencia con él, con sus prácticas. Desconfianza, tenían desconfianza en él".

El impacto que estos problemas generan en la orgánica del movimiento nos permite afirmar la importancia de la cohesión y el manejo de la conflictividad interna en los movimientos sociales territoriales.

\section{Manejo de la conflictividad interna}

Los movimientos sociales territoriales tienden a estar conformados por una amplia diversidad de sectores sociales (Asún \& Zúñiga, 2013). En los casos estudiados, participaron organizaciones sindicales, ambientalistas, vecinales, gremiales, empresariales y deportivas, compuestas por personas de distintas orientaciones políticas, nivel socioeconómico, experiencia, competencias, conocimientos e intereses. Por ello, la mantención de la cohesión interna fue una tarea crítica para ambos movimientos.

La dificultad de la tarea queda atestiguada en que en ambos casos estudiados la cohesión interna terminó por romperse. No obstante, los relatos de los dirigentes nos permiten reflexionar sobre qué tipos de capital podrian haber sido importantes para mantener la cohesión mientras duró cada movimiento, y cuáles podrían haber sido las carencias que llevaron a los quiebres.

En el MM, el capital cultural localizado y universalista de los dirigentes parece haber sido determinante. Éstos tenían certeza de que una de las principales estrategias que los gobiernos han utilizado para enfrentarse a los movimientos sociales es cooptar y/o dividir a sus dirigentes. Por ello, decidieron conducir el movimiento a través de una estructura asamblearia horizontal, en la que cada dirigente estaba en igualdad de condiciones y jerarquía, limitando la aparición de personalismos que pudieran luego ser cooptados. Además, en las negociaciones siempre estuvo presente gran parte de los dirigentes de la asamblea, lo que impedía que algunos de ellos se apartaran de los márgenes de negociación estipulados colectivamente. No obstante, ello no implica que todos participaran en la negociación de igual manera, es decir, con el mismo nivel de protagonismo.

Para controlar los conflictos internos producto de diferentes miradas sobre el movimiento o asimetrías de poder entre organizaciones, el capital cultural localizado orientó la implementación de estrategias y discursos específicos. Entre ellos, apelar a la identidad regional que los une como "patagónicos", por sobre las identidades grupales de cada orgánica, gremio o tendencia política. También el estar presentes en todas las protestas, entendiendo que la ciudadanía magallánica desconfiaba de quienes se mantienen solo en labores de representación o vocería.

Magallanes. E.1. "Hay regionalistas desde los comunistas, pasando por los demócrata-cristianos, por los socialistas, por los radicales, por la gente de derecha, por la UDI. Acá hay un regionalismo transversal". 
Magallanes. E.6. "El tema aquí es que se quiere a rabiar el regionalismo, el tema de la bandera magallánica es una cosa potente. (...) O sea, nos aprovechábamos del regionalismo que hay en nosotros y del tema del gas, que es una cosa muy, muy sensible para nosotros".

También fueron claves tanto el capital social fuerte de los dirigentes, en términos de confianza mutua, como su capital simbólico, que los llevaba a reconocerse mutuamente como representantes legítimos de distintas organizaciones ciudadanas, independiente de sus intereses y posturas políticas.

Magallanes. E.5. "Fui uno de los que propuso que la mesa debía ser una mesa horizontal, donde no existan celos dirigenciales, y que la representación legal estaba en las mismas organizaciones, porque cada una tenía su propia personalidad jurídica".

Magallanes. E.1. "Hay dirigentes de todos los partidos políticos y también numerosos dirigentes sociales que no son militantes de partidos políticos. Luego, [la Asamblea Ciudadana] es una red horizontal de organizaciones sociales, de dirigentes sociales y ciudadanos. No están ahí por su militancia política o partidaria, están ahí porque representan a una determinada organización social".

No obstante, la Asamblea Ciudadana de Magallanes se dividió luego de concluidas las negociaciones con el gobierno, producto del costo que tenía para los miembros de algunas organizaciones, principalmente transportistas, la mantención de la paralización regional, y las discrepancias ideológicas entre facciones del movimiento. Mientras los dirigentes sindicales y sociales proponían mantener la movilización para plantear otras demandas sensibles para la región, el sector ligado al mundo empresarial consideraba que aquello implicaba "politizar" una movilización netamente "ciudadana", acotada al tema del gas. Es de destacar, sin embargo, que la cohesión se mantuvo hasta el fin de las negociaciones con la autoridad nacional.

En el MA parece haber sido de igual importancia el capital cultural localizado para contener posibles deserciones, evitar confrontaciones entre dirigentes y organizaciones aliadas, priorizar aspectos compartidos, como el rechazo al centralismo y a las acciones del gobierno, y acentuar su sentido de identidad común, basado en la valoración del modo de vida local, la austeridad y un fuerte sentido familiar y comunitario.

Sin embargo, algunos dirigentes señalan que el movimiento no supo manejar las diferencias de estilo entre aquéllos de más edad, que tendían a una conducción más vertical y autoritaria, y los más jóvenes, provenientes principalmente del mundo estudiantil y ambientalista, con un estilo mucho más horizontal y asambleario. En este sentido, ni el conocimiento de los atributos de cada dirigencia y organización, ni la priorización de demandas y discursos aglutinantes, bastaron para evitar la emergencia de conflictividad.

Aysén. E.2. "Acá se hacían asambleas, pero eran muy, o sea... como te contaba, desde la visión antigua de organización. El dirigente ahí sentado y poco menos que enojado con nosotros porque le pediamos explicaciones de por qué estaban haciendo las cosas de esa forma".

Por otro lado, a diferencia del MM, los dirigentes del MA no pudieron establecer una asamblea que asegurase igualdad de voz y voto a todos los integrantes, lo que facilitó que el rol de representación y poder dentro del movimiento fuera concentrándose en uno o dos dirigentes, permitiendo que demandas cruciales para algunas organizaciones quedaran relegadas, aumentando la conflictividad interna. Por todo lo anterior, la confianza y el mutuo reconocimiento como pares legítimos, construidos en las etapas previas, se vieron debilitados, agotando las reservas de capital social fuerte y simbólico que había acumulado el movimiento.

Aysén. E.3. "Creo que el sistema de asambleas, donde la decisión la tome la gente, aunque se equivoque, no fue un ejercicio que se hizo mucho en este movimiento, a diferencia de los estudiantes o de Patagonia Sin Represas, donde todo se toma en asambleas. Los estudiantes se toman el tiempo, ellos se reúnen y ahí sacan un comunicado, y los voceros o los dirigentes son voceros. Aquí se le dio demasiado poder a un vocero, eran dos voceros, que tomó decisiones sin consultar al resto de la comunidad, y ahí hubo un tremendo error".

En suma, los dirigentes de ambos movimientos parecen haber tenido un alto nivel 
de capital cultural localizado y capital simbólico, que aportaron al diseño de las estrategias de interacción con el adversario y el manejo de la conflictividad interna. No obstante, el capital social fuerte y el capital simbólico de los dirigentes del MA se vieron debilitados simultáneamente. Las diferencias de estilo y criterio derivaron en desconfianzas y deslegitimación en la interna, y ello derivó en resultados menos satisfactorios para sus participantes, en comparación con el MM.

\section{CONCLUSIONES}

En este artículo hemos realizado un ejercicio teórico-empírico de explicación de la incidencia relativa de la movilización de distintos tipos de capital por parte de las dirigencias de dos movimientos sociales territoriales. Por supuesto, esta interpretación no es excluyente respecto de otras explicaciones, ni tampoco suponemos que la complementación de distintos tipos de capital es el único factor que influyó en la ocurrencia y el desarrollo de ambos conflictos. Por ejemplo, el hecho de que la protesta social esté cada vez más legitimada en Chile y el mundo como forma de participación política (Norris, 2002), puede haber facilitado la ocurrencia de ambos movimientos. Por otro lado, desde la teoría de las oportunidades políticas, la existencia de un gobierno de derecha en Chile puede haber tenido el mismo efecto. Asimismo, el auge de las protestas ecológico - territoriales en Chile (Delamaza et al. 2017) también puede dar luces sobre la emergencia de los movimientos aquí analizados y la manera en que se desarrollaron.

El relato de los dirigentes de ambos movimientos sociales territoriales respecto a su experiencia de movilización nos provee de información consistente para sostener la importancia de la posesión de distintos tipos de capital en los diversos procesos implicados en un movimiento social (Nepstad \& Bob, 2006). No obstante, nuestros resultados sugieren que pese a que los tres tipos de capital aquí analizados (cultural, social y simbólico) parecen ser herramientas necesarias para los dirigentes, algunos de estos capitales pueden haber sido más cruciales que otros en determinadas fases y desafíos del movimiento social.
La posesión de capital cultural localizado se posiciona como un factor de suma importancia en el desarrollo de estos movimientos sociales territoriales. En etapas iniciales (de emergencia del movimiento y búsqueda de adhesión de terceros), el conocimiento de características, valores, pautas culturales, comportamientos, estrategias históricas, trayectoria y experiencia de la población local y sus organizaciones, permite evaluar la correlación de fuerzas, cotejar fortalezas $y$ debilidades propias y así determinar la apertura (o clausura) de oportunidades políticas para la emergencia y crecimiento de una coalición. También permite identificar organizaciones sociales clave, y orientar estratégicamente la retórica del movimiento.

En las etapas posteriores, de confrontación con el adversario y manejo de la conflictividad interna, el capital cultural localizado permite orientar una serie de decisiones estratégicas: i) filtrar y acotar las demandas, distinguiendo lo posible, lo convocante, lo testimonial y lo estratégico; ii) acotar el repertorio de acciones colectivas, jerarquizándolas de acuerdo al contexto y las propias fortalezas y debilidades; iii) distribuir las tareas entre los dirigentes y miembros de cada organización; iv) generar una estructura organizacional lo suficientemente inclusiva y operativa como para movilizar de manera cohesionada a la coalición de organizaciones; y v) anticiparse al surgimiento de conflictividad interna y manejarla adecuadamente.

Por su parte, también pareciera haber jugado un rol estratégico en los movimientos estudiados la posesión de capital social fuerte entre los dirigentes. En los momentos iniciales de ambos ciclos de protestas, el que los dirigentes se hubieran conocido en movilizaciones previas posibilitó un rápido flujo de información, y la existencia de vínculos de confianza entre ellos, lo que a su vez permitió estructurar el movimiento de manera efectiva y expedita. Pero quizá incluso más importante fue el capital social fuerte durante la interacción con el adversario y la mantención de la cohesión interna, pues facilitó la división del trabajo y la mantención de la confianza que permitió a ambos movimientos resistir los primeros embates del gobierno.

En consecuencia, los resultados de este estudio permiten hipotetizar que el surgimiento y mantención de un movimiento social territorial 
dependen en gran medida de la buena comunicación y vínculos de confianza entre las organizaciones sociales del territorio, más aún si son anteriores a la emergencia del ciclo de protestas específico, y de que los dirigentes de cada una de ellas tengan un muy buen conocimiento de las características, habilidades, ideas y valores de su comunidad, de sus organizaciones sociales, sus militantes y dirigentes.

Lo anterior no significa que el resto de los capitales no sean relevantes. Por el contrario, el capital cultural universalista pareciera haber permitido comprender mejor las debilidades y fortalezas del adversario y sus estrategias de presión y negociación; el capital social débil facilitó el acceso a los medios de comunicación y al conocimiento técnico de otras organizaciones $y$ expertos que no participaban directamente en el movimiento o eran externos a la región; y el capital simbólico dio a los dirigentes la suficiente legitimidad interna para formar coaliciones, y legitimidad externa para mantener el apoyo de la ciudadanía. Además, encontramos que hay un tipo particular de conocimientos y habilidades referidos específicamente al trabajo activista, que tiene relación con la resistencia a las críticas internas, el manejo de demandas y otras tareas organizacionales en los movimientos sociales, que podemos entender como capital transcultural (Nepstad \& Bob, 2006), pero que otros autores han definido como "capital humano activista" (Van Dyke \& Dixon, 2013).

En síntesis, nuestros resultados sugieren que, aunque parece ser cierto que los dirigentes de un movimiento social requieren disponer de un "capital de liderazgo" integrado (Nepstad \& Bob, 2006) y que deben tener lazos fuertes y débiles con otras organizaciones (Ganz, 2010), no todos los tipos de capital son igualmente estratégicos en las distintas etapas del desarrollo de un movimiento social territorial.

De este modo, podrían destacarse dos aportes de esta investigación: por un lado, evidencia que el aparato teórico propuesto por Bourdieu puede ser de gran utilidad para el estudio del liderazgo en los movimientos sociales, incluso en un marco tan distinto a aquel en el que dicho modelo ha sido desarrollado. Por otro lado, evidencia que en un contexto en que la movilización se juega en lo territorial, el capital social fuerte y el capital cultural localizado pueden ser de especial relevancia. Es probable que, a escala local, donde muchas de las organizaciones convocadas son más bien informales, las confianzas interpersonales sean incluso más necesarias que en instancias en las que se cuenta con mayores niveles de institucionalización.

Respecto a las limitaciones del estudio, es de destacar que se ha estudiado un tipo de movimiento muy particular, como son los movimientos sociales territoriales, que apelan a una base de apoyo y a organizaciones mucho más heterogéneas que otros tipos de movimientos, por lo que es posible que los hallazgos de este estudio no sean pertinentes en otros tipos de movimientos. Además, se trató de dos casos relativamente similares: ambos movimientos se desarrollaron en regiones relativamente pequeñas en cuanto a población y aisladas del centro de poder, lo que puede haber facilitado y aumentado el rendimiento de algunos tipos de capitales de liderazgo sobre otros (como el capital cultural localizado y el social fuerte en comunidades de menor tamaño).

En función de lo anterior, consideramos de gran importancia continuar este ejercicio de aplicar las herramientas teóricas de Bourdieu (1986) a distintos tipos de contextos y movimientos sociales, utilizando distintos enfoques y métodos, para así adquirir mayor claridad respecto de los hallazgos que el presente estudio ha aportado.

\section{AGRADECIMIENTOS}

Este trabajo ha contado con financiamiento del Fondo Nacional de Desarrollo Científico y Tecnológico, Fondecyt, por medio de los proyectos 1140815 y 1160303.

\section{BIBLIOGRAFÍA}

Arenas, F. (2009). El Chile de las regiones: una historia inconclusa. Estudios Geográficos, 70(266), 11-39.

Asún, R., \& Zúñiga, C. (2013). ¿Por qué se participa? Explicando la protesta social regionalista a partir de dos Modelos psicosociales. Psicoperspectivas, 12(2), 38-50.

Ávila, R. (2002). Las relaciones entre la educación y la cultura en Pierre Bourdieu. Revista Colombiana de Sociología, 7(1), 9-26. 
Bennister, M., 't Hart, P., \& Worthy, B. (2014). Assessing the Authority of Political Office-Holders: The Leadership Capital Index. West European Politics, 38(3), 417440.

Bourdieu, P. (1980). El sentido Práctico. Madrid: Taurus.

Bourdieu, P. (1986). The forms of capital. En J. Richardson (Ed.) Handbook of Theory and Research for the Sociology of Education (241-258). New York: Greenwood.

Butorovic, N. (2015). Resumen meteorológico año 2014*

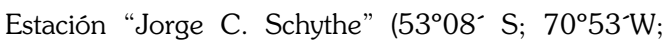
6 M.S.N.M). Anales del Instituto de la Patagonia, 43(1), 175-184.

Castón, P. (1996). La sociología de Pierre Bourdieu. Revista Española de Investigaciones Sociológicas 76, 75-98.

Cavallo, A., Salazar, M., \& Sepúlveda, O. (2008). La historia oculta del régimen militar: Chile 1973-1988. Santiago: Uqbar.

Consejo Nacional de la Cultura y las Artes (2015). Región de Aysén Síntesis regional. Recuperado de: http:// www.cultura.gob.cl/wp-content/uploads/2015/08/ Informe-Aysén-final.pdf

Crossley, N. (2003). From Reproduction to Transformation: Social Movement Fields and the Radical Habitus. Theory, Culture and Society, 20(6), 43-68.

Delamaza, G., Maillet, A., \& Neira, C. M. (2017). Socio-territorial conflicts in Chile: Configuration and politicization (2005-2014). European Review of Latin American and Caribbean Studies/Revista Europea de Estudios Latinoamericanos y del Caribe, (104), 23-46.

Diani, M. (1992). The Concept of Social Movement. The Sociological Review, 40(1), 1-25.

Fløysand, A., Barton, J., \& Román, Á. (2010). La doble jerarquía del desarrollo económico y gobierno local en Chile: El caso de la salmonicultura y los municipios chilotes. EURE, 36(108), 123-148.

Foldy, E., Goldman, L., \& Ospina, S. (2008). Sensegiving and the role of cognitive shifts in the work of leadership. The Leadership Quarterly, 19, 514-529.

Ganz, M. (2000). Resources and Resourcefulness: Strategic Capacity in the Unionization of California Agriculture, 1959-1966. American Journal of Sociology, 105(4), 1003-1062.

Ganz, M. (2010). Leading Change: Leadership, Organization, and Social Movements. En N. Nohria y R. Khurana (Eds.), Handbook of Leadership Theory and Practice (pp. 528-568). Boston: Harvard Business Press.

García, J. (2006). Educación y reproducción cultural: el Legado de Bourdieu. Práxis Educacional, 2(2), 71-77.

Gutiérrez, A. (2004). Poder, habitus y representaciones: recorrido por el concepto de violencia simbólica en Pierre Bourdieu. Revista Complutense de Educación, 15(1), 289-300.

Haluza-Delay, R. (2008). A theory of practice for social movements: Environmentalism and ecological habitus. Mobilization: The International Quarterly, 13(2), 205218. Husu, H. (2013). Bourdieu and Social Movements: Considering Identity Movements in Terms of Field, Capital and Habitus. Social Movement Studies, 12(3), 264-279.

Joignant, A. (2012). Habitus, campo y capital: Elementos para una teoría general del capital político. Revista Mexicana de Sociología, 74(4), 587-618.

Leupin, E. A. (2017). Las transiciones chilenas. Notas sobre discursos y representaciones acerca del proceso de transición a la democracia en Chile. Analele Universității Ovidius din Constanța-Seria Științe Politice, 6(1), 79-100.

McCarthy, J., \& Wolfson, M. (1996). Resource mobilization by local social movement organizations: Agency, strategy, and organization in the movement against drinking and driving. American Sociological Review, 61(6), 10701088

McCarthy, J., \& Zald, M. N. (2001). The Enduring Vitality of the Resource Mobilization Theory of Social Movements. En J. Turner (Ed.), Handbook of Sociological Theory (pp. 533-565). New York: Springer.

Ministerio de Desarrollo Social (2013). Resultados Regionales Encuesta de Caracterización Socioeconómica Nacional 2011: Región de Aysén. Recuperado de: http://observatorio.ministeriodesarrollosocial.gob.cl/ layout/doc/casen/XI_Aysen_oct13.pdf

Morris, A., \& Staggenborg, S. (2004). Leadership in Social Movements. En D. Snow, S. Soule y H. Kriesi (Eds.), The Blackwell Companion to Social Movements (pp. 171-196). Oxford: Blackwell Publishing Ltd.

Nepstad, S., \& Bob, C. (2006). When do leaders matter? Hypotheses on leadership dynamics in social movements. Mobilization: An International Quarterly, 11(1), 1-22.

Norris, P. (2002). Democratic Phoenix. Cambridge: Cambridge University Press.

Radovic, N. (2013). La movilización colectiva de la Patagonia norte de Chile: un enfoque etnográfico de las demandas de la región de Aysén, 2012. Trabajo presentado en VII Jornadas Santiago Wallace de Investigación en Antropología Social, Buenos Aires. Resumen recuperado de: https://www.aacademica. org/000-063/525 
Rizo, M. (2006). Conceptos para pensar lo urbano: el abordaje de la ciudad desde la identidad, el habitus y las representaciones sociales. Bifurcaciones, 6, 1-13.

Robinson, E. (2009). Mobilizing voices: A discussion of leadership in an environmentally contaminated community. Qualitative Sociology Review, 5(1), 70-97.

Salazar, G. (2005). Construcción de Estado en Chile. Santiago de Chile: Sudamericana.

Smith, J., \& Fetner, T. (2008). Structural Approaches in the Study of Social Movements. En B. Klandermans y C. Roggeband (Eds.), Handbook of Social Movements:
Social Movements Across Disciplines (pp. 13-58). New York: Springer.

Snow, D., Benford R., McCammon, H., Hewitt, L., \& Fitzgerald, S. (2014). The emergence, development, and future of the framing perspective: 25 years since "Frame alignment". Mobilization: An International Quarterly, 19(1), 23-46.

Van Dyke, N., \& Dixon, M. (2013). Activist human capital: skills acquisition and the development of commitment to social movement activism. Mobilization: An International Journal 18(2): 197-212. 